\title{
Viscosity and density measurements of aqueous amines at high pressures: MDEA-water and MEA-water mixtures for $\mathrm{CO}_{2}$ capture
}

Manuel Sobrino, Eduardo I. Concepción, Ángel Gómez-Hernández, M. Carmen Martín, José J. Segovia*

TERMOCAL Research Group, Escuela de Ingenierías Industriales, Universidad de Valladolid, Paseo del Cauce 59, 47011 Valladolid, Spain.

*corresponding autor, e-mail: jose.segovia@eii.uva.es

\section{ABSTRACT}

Viscosity and density are thermophysical properties crucial to characterizing any kind of fluid such as aqueous amines. These blends are becoming more and more relevant for their $\mathrm{CO}_{2}$ capture potential, such that having accurate viscosity and density measurements would prove useful. Densities and viscosities of these mixtures at atmospheric pressure may be found in the literature although it is more difficult to find values at high pressures, these potentially proving interesting when seeking to provide a full description of these fluids.

Viscosity and density measurements at high pressures (up to $120 \mathrm{MPa}$ ) and at temperatures between 293.15 and 353.15 K of MDEA + water and MEA + water mixtures (both from $10 \%$ to $40 \%$ amine mass fraction) are presented in this work. Density measurements were performed with an Anton Paar DMA HPM densimeter with an expanded uncertainty $(k=2)$ less than $\pm 0.7 \mathrm{~kg} \cdot \mathrm{m}^{-3}$. A falling body technique was used to measure viscosities at high pressures due to its sturdiness in terms of corrosion. Details of this latter equipment are presented, including calibration using $n$-dodecane and uncertainty calculations, which give a relative expanded uncertainty $(k=2)$ of less than $\pm 2.4 \%$ for the highest viscosity and \pm 2.9 $\%$ for the lowest. 
Keywords: Viscosity; Density; Falling body viscometer; High pressure; MDEA; MEA.

\section{Introduction}

Society is becoming increasingly aware of environmental issues, with the focus on problems such as the greenhouse effect. In this sense, reducing $\mathrm{CO}_{2}$ emissions is an important goal. Although there are many substances whose Global Warming Potential (GWP) is higher than the GWP of $\mathrm{CO}_{2}$, our current way of life, which is mainly based on fossil fuels, forces us to pay particular attention to $\mathrm{CO}_{2}$ emissions.

There are two main actions which might help to improve this situation. Firstly, an increased use of renewable sources will reduce $\mathrm{CO}_{2}$ emissions directly, and secondly, $\mathrm{CO}_{2}$ may be removed from gas streams by using substances such as aqueous amines. In this regard, many initiatives have been promoted by governments over the last few years in an effort to cut $\mathrm{CO}_{2}$ emissions.

Specifically, alkanolamine solutions are widely used in industry to remove components such as $\mathrm{H}_{2} \mathrm{~S}$ and $\mathrm{CO}_{2}$ from natural or refinery gases, with the tertiary amine $n$ methyldiethanolamine (MDEA) an industrially important one for this purpose $[1,2]$. Primary amine monoethanolamine (MEA) is one of the most effective amines for $\mathrm{CO}_{2}$ absorption, reaching efficiency rates above $90 \%$ [3] and being catalogued as one of the most promising amines for these purposes by Aaron et al. [4].

Thermophysical properties such as viscosity and density of aqueous solutions are required for two main reasons. Firstly, they are crucial for designing treatment equipment [5], and secondly, knowledge of these properties, even at high pressures, will enable a full characterization of these fluids.

In this work, viscosity and density measurements of MDEA $+\mathrm{H}_{2} \mathrm{O}$ and MEA $+\mathrm{H}_{2} \mathrm{O}$ mixtures $(10 \%, 20 \%, 30 \%$ and $40 \%$ amine mass fraction) at pressures from 0.1 to $120 \mathrm{MPa}$ and 
temperatures of $293.15,313.15,333.15$ and $353.15 \mathrm{~K}$ are presented. Density measurements were carried out with an Anton Paar DMA HPM densimeter, already introduced [6], and density measurements were extended up to $p=140 \mathrm{MPa}$ and $T=393.15 \mathrm{~K}$. Viscosity measurements were performed with a falling body viscometer recently developed at the TERMOCAL laboratory [7]. Both techniques are able to resist any corrosion effects which might be caused by amines.

\section{Experimental procedure}

\subsection{Densimeter}

An Anton Paar DMA HPM densimeter was employed to perform the density measurements using water and vacuum for its calibration and following the method shown in [6]. Uncertainty calculations were carried out following the procedure described in JCGM 100:2008 [8] and explained in [6], obtaining an expanded uncertainty $(k=2)$ of below \pm 0.7 $\mathrm{kg} \cdot \mathrm{m}^{-3}$

\subsection{Viscometer}

A falling body viscometer was used for the measurements. It is based on the falling time measurement of a body (a cylinder in our case) when it falls through a vertical pipe containing the fluid whose viscosity we wish to know. The apparatus is able to measure in wide pressure ranges, from $0.1 \mathrm{MPa}$ to $140 \mathrm{MPa}$, and temperature, from $253.15 \mathrm{~K}$ to $523.15 \mathrm{~K}$.

The cell, where measurements take place, was designed by the Groupe de Haute Pression, Laboratoire des Fluides Complexes of the University of Pau [9], and its full experimental setup was developed and improved at the TERMOCAL laboratory.

Considering that the body reaches its terminal velocity without eccentricity and laminar flow, equation (1) could theoretically describe the behaviour of this sort of viscometers. This expression is based on the Stokes' law and Newton's second law: 
$\eta=K \cdot \Delta \rho \cdot \Delta t$

The terms of the expression (1) are: $\eta$ the viscosity, $K$ a calibration constant which depends on the apparatus and the falling body, $\Delta \rho$ the difference between the density of the body material and the liquid density, and $\Delta t$ the time registered between two coils.

In an ideal case, $K$ could be determined without any calibration from the dimensions of the instrument, the mass of the falling cylinder and its density using a mathematical expression. However, in practice, this is not advisable because real operation of this instrument differs from the simplified model given by that mathematical expression in several factors $[10,11]$, which is why a calibration procedure is always performed in this kind of viscometer. Several ways of calibration based on equation (1) have been successfully performed [12]: from the use of a single calibration constant modified by thermal expansion coefficients to using several calibration constants for each temperature and pressure set.

In our case, the model described by equation (1) fits the range of viscosities considering in this work (up to $5 \mathrm{mPa} \cdot \mathrm{s}$ approximately) quite well. However, adding an independent term (intercept) to the expression (1) has allowed us to achieve a better approach to the behaviour of our viscometer. Expression (2) is therefore used in this work:

$\eta=a+b \cdot \Delta t \cdot \Delta \rho$

Although this expression has already been used by other authors, it is important to highlight how it is used. As will be shown in the calibration procedure, equation (2) would be applied regardless temperature and pressure. This offers a substantial advantage since we can measure at any temperature and pressure condition with a single fitting, as long as measured viscosities are within its calibration range.

The experimental setup was developed in full at the TERMOCAL laboratory using high pressure equipment and was described in [13]. The principal elements are the measuring cell, a rotary valve which allows us to rotate the cell automatically, a thermostatic bath (Julabo 
F81-ME), a pressure generator (HiP 50-5.75-30), a digital gauge (Druck DPI 104), a vacuum pump (Leybold TRIVAC D8B) with a cold trap, and the electronic devices: $16 \mathrm{Ch}$. Multifunction Data Acquisition Unit 16 bits (Agilent U2352A), Data Acquisition Unit for the temperature (Agilent 34970A), Arbitrary Waveform Generator (Agilent, 33220A). In addition, resistance thermometers attached to the coils were installed to control temperature and another pressure generator (HiP 50-6-15) linked to an engine was added to control the pressure automatically.

The measuring cell, which is the core of this viscometer, is presented in Figure 1. It has two concentric tubes whose length is $400 \mathrm{~mm}$ and their diameters are $6.52 \mathrm{~mm}$ and $8.1 \mathrm{~mm}$. Both of them are filled with the pressurized liquid, maintaining the same pressure inside and outside the inner tube and avoiding any risk of deformation. In addition, four coils, spaced 50 $\mathrm{mm}$ apart, are arranged around the tube. Those coils are placed towards the bottom so as to favour terminal velocity being reached. It has been proved in a previous work [14] that terminal velocity is reached in all the cases. Thus, in order to avoid signal interferences between coils, the two intermediate coils are disconnected, and the time from the first to the fourth coil (separated by $150 \mathrm{~mm}$ ) is taken. Tubes and coils are surrounded by a thermostatic fluid which flows from the bath. The falling cylinder (shown in Figure 1) is made of magnetic stainless steel with a hemispherical end and has a length of $20 \mathrm{~mm}$ and a diameter of 6.20 $\mathrm{mm}$. The density of the body (approximately constant) is determined using a pycnometer $\rho=$ $(7.673 \pm 0.017) \mathrm{g} \cdot \mathrm{cm}^{-3}$. The relationship between the diameter of the inner tube and the diameter of the falling body is 0.951 . This value is higher than the critical value of 0.93 established by Chen et al. [15] and also higher than the more conservative value of 0.95 established by Vant and used by Schaschke et al. [16] and Zeng et al. [11]. Working below those values could cause undesirable eccentricity effects. 


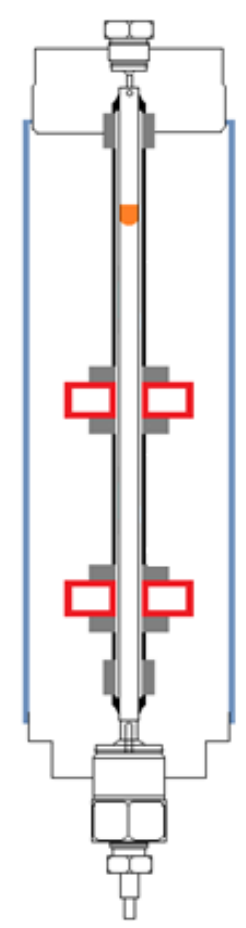

Figure 1. Measuring cell with two operating coils.

A time measurement system was designed and described previously $[7,17]$ and allows time to be measured with an expanded uncertainty $(k=2)$ of $\pm 0.01 \mathrm{~s}$.

A computer program using Agilent VEE Pro software was developed in full at the TERMOCAL laboratory to record all the parameters involved in our measurements (falling time, pressure, temperature...). This time measurement system is an important improvement for such falling body techniques and provide accurate time measurements which implies accurate viscosities.

\subsection{Materials}

The materials used in the calibration and the measurements were used without further purification and their purity was checked by gas chromatography. Table 1 summarizes their characteristics. 
Table 1. Material description.

\begin{tabular}{llccc}
\hline Compound & \multicolumn{1}{c}{ Source } & $\begin{array}{c}\text { Mass fraction } \\
\text { purity }\end{array}$ & Water content (\%) & $\begin{array}{c}\text { Purification } \\
\text { method }\end{array}$ \\
\hline$n$-Dodecane & Sigma-Aldrich & $\geq 0.99$ & Máx. 0.01 & None \\
1-Butanol & Sigma-Aldrich & $\geq 0.995$ & $(157.5 \mathrm{ppm})$ & None \\
MDEA & Aldrich Chemistry & $\geq 0.999$ & Máx. 0.1 & None \\
MEA & Sigma-Aldrich & $\geq 0.998$ & Máx. 0.14 & None \\
Water & Sigma-Aldrich & Conductivity $\leq 2 \cdot 10^{-6} \mathrm{ohm}^{-1} \cdot \mathrm{cm}^{-1}$ & None \\
\hline
\end{tabular}

${ }^{a}$ As stated by the supplier and checked by gas chromatography.

2.4 Viscometer calibration

Calibration of the falling body viscometer was performed from $0.1 \mathrm{MPa}$ to $120 \mathrm{MPa}$ and from 293.15 $\mathrm{K}$ to $353.15 \mathrm{~K}$ with $n$-dodecane as calibration fluid and the correlation proposed by Caudwell et al. [18] was used. It is important to highlight that Caudwell et al. [18] correlation begins at $298.15 \mathrm{~K}$, so data for calibration at $293.15 \mathrm{~K}$ were also taken from [13], both data based on vibrating wire viscometer measurements.

Fall time was recorded considering fifteen repetitions for each pressure and temperature. After that, calibration consists of fitting all points (figure 2) using the model expressed by equation (2), whose R-squared value indicates that the model defined by the parameters given in table 2 explains $99.89 \%$ of variability. 


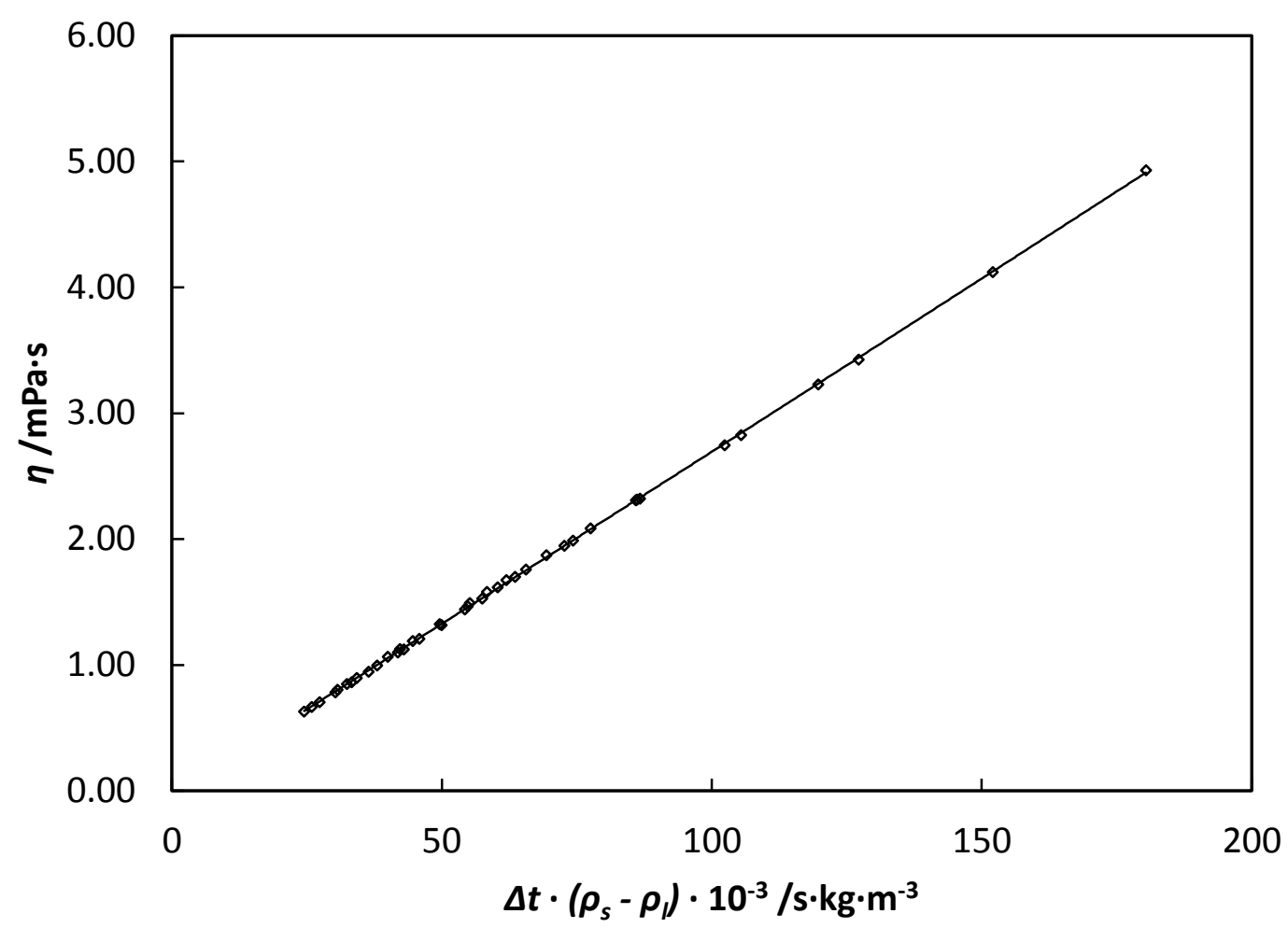

Figure 2. Falling body viscometer calibration fitting using $n$-dodecane as reference fluid.

Table 2. Coefficients of equation (2) obtained for the falling body viscometer calibration with n-dodecane.

\begin{tabular}{ccc}
\hline & Parameters & Standard deviation \\
\hline $\mathrm{a} / \mathrm{mPa} \cdot \mathrm{s}$ & $-4.49383 \cdot 10^{-2}$ & $8.7 \cdot 10^{-3}$ \\
$\mathrm{~b} / \mathrm{mPa} \cdot \mathrm{m}^{3} \cdot \mathrm{kg}^{-1}$ & $2.77769 \cdot 10^{-5}$ & $1.1 \cdot 10^{-7}$ \\
\hline
\end{tabular}

According to the procedure described, the range of the present calibration is from $\eta=0.630$ $\mathrm{mPa} \cdot \mathrm{s}(n$-dodecane at $T=353.15 \mathrm{~K}$ and $p=0.1 \mathrm{MPa})$ to $\eta=4.929 \mathrm{mPa} \cdot \mathrm{s}(n$-dodecane at $T=$ 293.15 K and $p=120 \mathrm{MPa})$. 


\subsection{Uncertainty calculation for viscosity}

Uncertainty calculation was carried out following the model expressed by equation (2) and the procedure described in JCGM 100:2008 [8] and its results are presented in tables 3 and 4 [17]. Uncertainty was evaluated at the limits of the calibration viscosity range for the studied mixtures, whose results are presented in the next section: the lowest viscosity $(0.617 \mathrm{mPa} \cdot \mathrm{s}$, MDEA $20 \%+\mathrm{H}_{2} \mathrm{O}$ at $T=353.15 \mathrm{~K}$ and $\left.p=0.1 \mathrm{MPa}\right)$ and the highest viscosity (4.954 $\mathrm{mPa} \cdot \mathrm{s}$, MEA $40 \%+\mathrm{H}_{2} \mathrm{O}$ at $T=293.15 \mathrm{~K}$ and $p=120 \mathrm{MPa}$ ). It has been considered a normal distribution with a coverage factor $k=2$ (confidence level of $95.45 \%$ ), obtaining a relative expanded uncertainty which varies from $\pm 2.4 \%$ to $\pm 2.9 \%$ for the highest and lowest viscosities, respectively. It is interesting to highlight that the most significant contribution in both cases is the uncertainty associated to calibration function coefficients. 
Table 3. Uncertainty calculation of dynamic viscosity, $\eta$, for MEA (1) $+\mathrm{H}_{2} \mathrm{O}(2)\left(w_{1}=0.4001\right)$ at $p=120 \mathrm{MPa}$ and $T=293.15 \mathrm{~K}$.

\begin{tabular}{|c|c|c|c|c|c|c|c|}
\hline Amount & & Estimate & Units & Probability & Standard & $\begin{array}{c}\text { Coefficient } \\
\text { of }\end{array}$ & $\begin{array}{c}\text { Contribution } \\
\text { to }\end{array}$ \\
\hline & & & & & & Sensitivity & Uncertainty \\
\hline$X_{i}$ & & $x_{i}$ & & & $u\left(x_{i}\right)$ & $c_{i}$ & $u(y)$ \\
\hline Reference & & & & & & & \\
\hline Fluid & Viscosity & 4.954 & $\mathrm{mPa} \cdot \mathrm{s}$ & Normal & 0.050 & 1 & 0.050 \\
\hline & Calibration & & $\mathrm{s}$ & Normal & 0.005 & 0.19 & 0.0010 \\
\hline Time & Resolution & 27.20 & $\mathrm{~s}$ & Rectangular & 0.0029 & 0.19 & 0.00056 \\
\hline & Repeatability & & $\mathrm{s}$ & Normal & 0.070 & 0.19 & 0.014 \\
\hline & Calibration & & $\mathrm{K}$ & Normal & 0.010 & 0.085 & 0.00085 \\
\hline & Resolution & 293.15 & $\mathrm{~K}$ & Rectangular & 0.0029 & 0.085 & 0.00025 \\
\hline 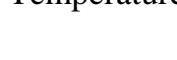 & Uniformity & & K & Rectangular & 0.029 & 0.085 & 0.0025 \\
\hline & Stability & & $\mathrm{K}$ & Rectangular & 0.014 & 0.085 & 0.0012 \\
\hline & Calibration & & $\mathrm{MPa}$ & Normal & 0.00001 & 0.040 & 0.0000 \\
\hline Pressure & Resolution & 120 & $\mathrm{MPa}$ & Rectangular & 0.0029 & 0.040 & 0.00012 \\
\hline & Stability & & $\mathrm{MPa}$ & Rectangular & 0.014 & 0.040 & 0.00058 \\
\hline & Solid & 7673 & $\mathrm{~kg} \cdot \mathrm{m}^{-3}$ & Normal & 17 & 0.00073 & 0.012 \\
\hline & Fluid & 1056.3 & $\mathrm{~kg} \cdot \mathrm{m}^{-3}$ & Normal & 0.42 & 0.00073 & 0.00031 \\
\hline Calibration & & & & & & & \\
\hline function & & & $\mathrm{mPa} \cdot \mathrm{s}$ & Normal & 0.030 & 1 & 0.030 \\
\hline coefficients & & & & & & & \\
\hline Standard Un & rtainty & & $\mathrm{mPa} \cdot \mathrm{s}$ & & & $u(y)$ & \pm 0.061 \\
\hline Expanded U & ertainty $(k=2)$ & & $\mathrm{mPa} \cdot \mathrm{s}$ & & & $U(y)$ & \pm 0.12 \\
\hline Relative $\mathrm{Ex}_{1}$ & ded Uncertaint & $(k=2)$ & $(\mathrm{mPa} \cdot \mathrm{s} / \mathrm{m}$ & & & $U_{r}(y)$ & $\pm \mathbf{0 . 0 2 4}$ \\
\hline
\end{tabular}


Table 4. Uncertainty calculation of dynamic viscosity, $\eta$, for MDEA (1) $+\mathrm{H}_{2} \mathrm{O}(2)\left(w_{1}=\right.$ $0.2002)$ at $p=0.1 \mathrm{MPa}$ and $T=353.15 \mathrm{~K}$.

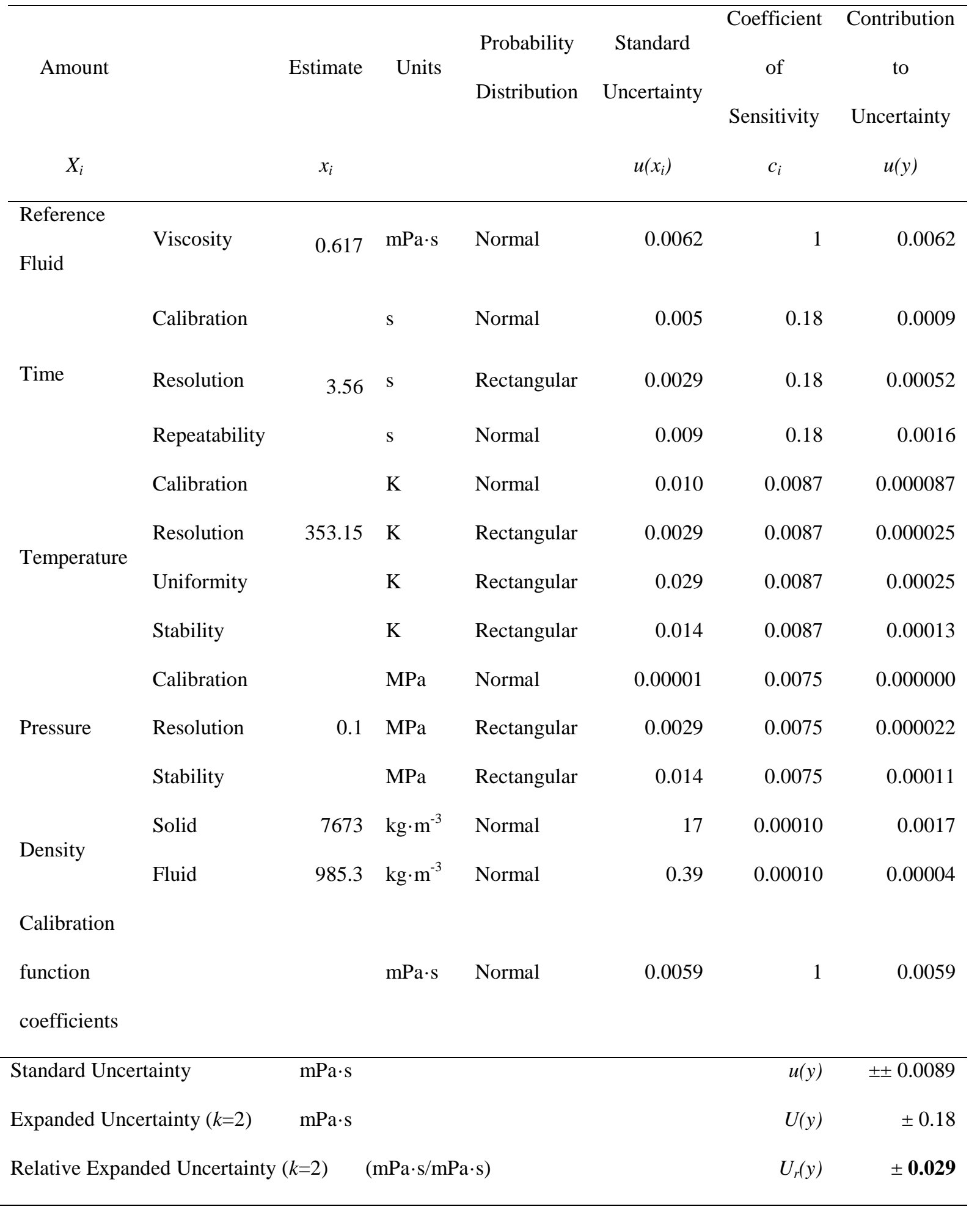




\section{Results and discussion}

Density measurements of aqueous solutions of methyldiethanolamine (MDEA) and monoethanolamina (MEA) were performed at pressures from 0.1 to $140 \mathrm{MPa}$ for MDEA and up to $120 \mathrm{MPa}$ for MEA and six temperatures from 293.15 to $393.15 \mathrm{~K}$ for different amine mass fractions: $0.1,0.2,0.3$ and 0.4 . These experimental results are summarized in table 5 for MDEA and table 6 for MEA.

Table 5. Experimental densities, $\rho$, for MDEA (1) $+\mathrm{H}_{2} \mathrm{O}(2)$ mixtures at different conditions of temperature, $T$, pressure, $p$, and mass fraction, $w_{1}{ }^{\mathrm{a}}$

\begin{tabular}{rcccccc}
\hline & \multicolumn{5}{c}{$\rho / \mathrm{kg} \cdot \mathrm{m}^{-3}$} \\
\cline { 2 - 7 }$p / \mathrm{MPa}$ & 293.15 & 313.15 & 333.15 & 353.15 & 373.15 & 393.15 \\
\hline 0.1 & 1007.0 & 1000.2 & 990.5 & 978.6 & 964.4 & 948.6 \\
0.5 & 1007.2 & 1000.4 & 990.6 & 978.7 & 964.6 & 949.0 \\
1 & 1007.4 & 1000.6 & 990.8 & 978.9 & 964.9 & 949.2 \\
2 & 1007.8 & 1001.0 & 991.3 & 979.3 & 965.3 & 949.6 \\
5 & 1009.0 & 1002.2 & 992.5 & 980.6 & 966.7 & 951.1 \\
10 & 1011.1 & 1004.3 & 994.6 & 982.8 & 969.0 & 953.6 \\
15 & 1013.2 & 1006.3 & 996.6 & 984.9 & 971.2 & 956.0 \\
20 & 1015.3 & 1008.2 & 998.6 & 987.0 & 973.5 & 958.5 \\
30 & 1019.4 & 1012.3 & 1002.6 & 991.2 & 977.9 & 963.0 \\
40 & 1023.4 & 1016.2 & 1006.7 & 995.2 & 982.1 & 967.6 \\
50 & 1027.3 & 1019.9 & 1010.4 & 999.1 & 986.2 & 971.8 \\
60 & 1031.1 & 1023.8 & 1014.2 & 1003.1 & 990.1 & 976.2 \\
70 & 1035.0 & 1027.4 & 1017.9 & 1006.9 & 994.2 & 980.4 \\
& 1038.5 & 1031.0 & 1021.6 & 1010.5 & 998.1 & 984.3
\end{tabular}




\begin{tabular}{|c|c|c|c|c|c|c|}
\hline 90 & 1042.2 & 1034.5 & 1025.1 & 1014.0 & 1001.8 & 988.3 \\
\hline 100 & 1045.7 & 1038.2 & 1028.5 & 1017.7 & 1005.3 & 992.1 \\
\hline 110 & 1049.2 & 1041.5 & 1031.9 & 1021.2 & 1009.0 & 995.9 \\
\hline 120 & 1052.7 & 1044.9 & 1035.4 & 1024.6 & 1012.6 & 999.6 \\
\hline 130 & 1056.1 & 1048.1 & 1038.7 & 1028.0 & 1016.0 & 1003.1 \\
\hline 140 & 1059.5 & 1051.4 & 1042.0 & 1031.2 & 1019.4 & 1006.7 \\
\hline \multicolumn{7}{|c|}{$w_{1}=0.2002$} \\
\hline 0.1 & 1016.5 & 1008.5 & 997.8 & 985.3 & 970.5 & 954.0 \\
\hline 0.5 & 1016.6 & 1008.6 & 998.0 & 985.3 & 970.7 & 954.4 \\
\hline 1 & 1016.8 & 1008.8 & 998.2 & 985.5 & 970.9 & 954.6 \\
\hline 2 & 1017.2 & 1009.2 & 998.6 & 985.9 & 971.4 & 955.2 \\
\hline 5 & 1018.4 & 1010.4 & 999.8 & 987.2 & 972.8 & 956.7 \\
\hline 10 & 1020.3 & 1012.3 & 1001.8 & 989.4 & 975.0 & 959.2 \\
\hline 15 & 1022.2 & 1014.2 & 1003.8 & 991.5 & 977.2 & 961.6 \\
\hline 20 & 1024.2 & 1016.1 & 1005.7 & 993.5 & 979.5 & 964.0 \\
\hline 30 & 1028.0 & 1019.9 & 1009.7 & 997.6 & 983.9 & 968.5 \\
\hline 40 & 1031.8 & 1023.7 & 1013.6 & 1001.6 & 988.2 & 973.1 \\
\hline 50 & 1035.4 & 1027.3 & 1017.2 & 1005.5 & 992.1 & 977.3 \\
\hline 60 & 1039.0 & 1031.0 & 1020.9 & 1009.3 & 996.1 & 981.7 \\
\hline 70 & 1042.6 & 1034.4 & 1024.4 & 1012.9 & 1000.0 & 985.9 \\
\hline 80 & 1045.9 & 1037.9 & 1027.9 & 1016.6 & 1003.8 & 989.8 \\
\hline 90 & 1049.4 & 1041.2 & 1031.3 & 1020.0 & 1007.4 & 993.7 \\
\hline 100 & 1052.8 & 1044.7 & 1034.7 & 1023.6 & 1011.1 & 997.5 \\
\hline 110 & 1056.0 & 1047.9 & 1038.0 & 1027.0 & 1014.7 & 1001.4 \\
\hline 120 & 1059.3 & 1051.1 & 1041.4 & 1030.4 & 1018.3 & 1005.0 \\
\hline 130 & 1062.6 & 1054.3 & 1044.5 & 1033.6 & 1021.5 & 1008.5 \\
\hline 140 & 1065.6 & 1057.4 & 1047.7 & 1036.8 & 1025.0 & 1012.0 \\
\hline & & & $w_{1}=$ & & & \\
\hline
\end{tabular}




\begin{tabular}{|c|c|c|c|c|c|c|}
\hline 0.1 & 1026.7 & 1017.2 & 1005.4 & 992.0 & 976.5 & 959.6 \\
\hline 0.5 & 1026.9 & 1017.4 & 1005.6 & 992.0 & 976.6 & 959.9 \\
\hline 1 & 1027.1 & 1017.5 & 1005.7 & 992.2 & 976.9 & 960.1 \\
\hline 2 & 1027.4 & 1017.9 & 1006.2 & 992.7 & 977.4 & 960.6 \\
\hline 5 & 1028.6 & 1019.1 & 1007.3 & 993.9 & 978.7 & 962.1 \\
\hline 10 & 1030.4 & 1020.9 & 1009.3 & 996.1 & 981.1 & 964.7 \\
\hline 15 & 1032.2 & 1022.8 & 1011.3 & 998.2 & 983.4 & 967.1 \\
\hline 20 & 1034.1 & 1024.6 & 1013.2 & 1000.3 & 985.6 & 969.6 \\
\hline 30 & 1037.7 & 1028.4 & 1017.0 & 1004.3 & 989.8 & 974.2 \\
\hline 40 & 1041.2 & 1032.0 & 1020.9 & 1008.1 & 994.1 & 978.7 \\
\hline 50 & 1044.6 & 1035.5 & 1024.3 & 1012.0 & 998.1 & 983.0 \\
\hline 60 & 1048.0 & 1039.0 & 1027.9 & 1015.9 & 1002.1 & 987.4 \\
\hline 70 & 1051.4 & 1042.3 & 1031.5 & 1019.5 & 1005.9 & 991.6 \\
\hline 80 & 1054.6 & 1045.5 & 1034.9 & 1023.0 & 1009.8 & 995.4 \\
\hline 90 & 1057.9 & 1048.7 & 1038.1 & 1026.4 & 1013.3 & 999.4 \\
\hline 100 & 1061.1 & 1052.0 & 1041.6 & 1029.9 & 1017.0 & 1003.1 \\
\hline 110 & 1064.1 & 1055.2 & 1044.6 & 1033.3 & 1020.5 & 1006.9 \\
\hline 120 & 1067.2 & 1058.3 & 1047.9 & 1036.6 & 1024.0 & 1010.6 \\
\hline 130 & 1070.3 & 1061.3 & 1051.0 & 1039.8 & 1027.4 & 1014.1 \\
\hline 140 & 1073.2 & 1064.5 & 1054.2 & 1042.9 & 1030.7 & 1017.6 \\
\hline & \multicolumn{6}{|c|}{$w_{1}=0.4000$} \\
\hline 0.1 & 1036.6 & 1025.5 & 1012.4 & 998.1 & 981.9 & 964.2 \\
\hline 0.5 & 1036.8 & 1025.6 & 1012.7 & 998.2 & 982.1 & 964.7 \\
\hline 1 & 1036.9 & 1025.8 & 1012.8 & 998.4 & 982.4 & 964.8 \\
\hline 2 & 1037.3 & 1026.2 & 1013.2 & 998.8 & 982.8 & 965.4 \\
\hline 5 & 1038.3 & 1027.3 & 1014.4 & 1000.1 & 984.2 & 966.9 \\
\hline 10 & 1040.1 & 1029.2 & 1016.4 & 1002.3 & 986.5 & 969.5 \\
\hline
\end{tabular}




\begin{tabular}{lllllll}
15 & 1041.9 & 1031.0 & 1018.4 & 1004.4 & 988.8 & 972.0 \\
20 & 1043.6 & 1032.8 & 1020.3 & 1006.5 & 991.1 & 974.5 \\
30 & 1047.1 & 1036.3 & 1024.1 & 1010.5 & 995.5 & 979.2 \\
40 & 1050.4 & 1040.0 & 1027.9 & 1014.4 & 999.8 & 983.9 \\
50 & 1053.8 & 1043.2 & 1031.4 & 1018.2 & 1003.7 & 988.1 \\
60 & 1057.1 & 1046.6 & 1035.0 & 1022.0 & 1007.7 & 992.5 \\
70 & 1060.3 & 1050.0 & 1038.4 & 1025.6 & 1011.6 & 996.8 \\
80 & 1063.3 & 1053.1 & 1041.6 & 1029.1 & 1015.4 & 1000.8 \\
90 & 1066.4 & 1056.2 & 1044.9 & 1032.6 & 1019.0 & 1004.6 \\
100 & 1069.5 & 1059.5 & 1048.2 & 1036.0 & 1022.6 & 1008.5 \\
110 & 1072.4 & 1062.5 & 1051.3 & 1039.3 & 1026.2 & 1012.3 \\
120 & 1075.4 & 1065.5 & 1054.5 & 1042.5 & 1029.7 & 1015.9 \\
130 & 1078.4 & 1068.4 & 1057.5 & 1045.8 & 1033.0 & 1019.4 \\
140 & 1081.1 & 1071.4 & 1060.6 & 1048.9 & 1036.3 & 1023.0 \\
\hline
\end{tabular}

${ }^{\mathrm{a}}$ Standard uncertainties $u$ are: $u(T)=0.01 \mathrm{~K} ; u_{\mathrm{r}}(p)=0.0001(\mathrm{kPa} / \mathrm{kPa}) ; u(w)=0.0001$ and $u(\rho)=0.35 \mathrm{~kg} \cdot \mathrm{m}^{-3}$

Table 6. Experimental densities, $\rho$, for MEA (1) $+\mathrm{H}_{2} \mathrm{O}(2)$ mixtures at different conditions of temperature, $T$, pressure, $p$, and mass fraction, $w_{1}{ }^{\mathrm{a}}$

\begin{tabular}{rrrrrrr}
\hline & \multicolumn{5}{c}{$\rho / \mathrm{kg} \cdot \mathrm{m}^{-3}$} \\
\cline { 3 - 7 }$p / \mathrm{MPa}$ & 293.15 & 313.15 & 333.15 & 353.15 & 373.15 & 393.15 \\
\hline 0.1 & 1002.5 & 995.9 & 986.3 & 974.7 & 960.8 & 945.2 \\
0.5 & 1002.7 & 996.0 & 986.5 & 974.7 & 961.0 & 945.6 \\
1 & 1002.9 & 996.3 & 986.7 & 975.0 & 961.3 & 945.8 \\
2 & 1003.3 & 996.6 & 987.1 & 975.4 & 961.8 & 946.3 \\
5 & 1004.6 & 997.9 & 988.4 & 976.7 & 963.1 & 947.8
\end{tabular}




\begin{tabular}{|c|c|c|c|c|c|c|}
\hline 10 & 1006.7 & 1000.0 & 990.5 & 978.9 & 965.5 & 950.3 \\
\hline 15 & 1008.8 & 1002.0 & 992.6 & 981.1 & 967.7 & 952.7 \\
\hline 20 & 1010.9 & 1004.0 & 994.6 & 983.2 & 969.9 & 955.2 \\
\hline 30 & 1015.1 & 1008.1 & 998.6 & 987.4 & 974.4 & 959.8 \\
\hline 40 & 1019.1 & 1012.1 & 1002.7 & 991.4 & 978.7 & 964.4 \\
\hline 50 & 1023.0 & 1015.9 & 1006.5 & 995.4 & 982.8 & 968.7 \\
\hline 60 & 1026.9 & 1019.8 & 1010.4 & 999.4 & 986.8 & 973.0 \\
\hline 70 & 1030.8 & 1023.4 & 1014.1 & 1003.1 & 990.8 & 977.3 \\
\hline 80 & 1034.5 & 1027.0 & 1017.7 & 1006.9 & 994.8 & 981.3 \\
\hline 90 & 1038.2 & 1030.6 & 1021.3 & 1010.5 & 998.5 & 985.2 \\
\hline 100 & 1041.8 & 1034.2 & 1024.8 & 1014.1 & 1002.2 & 989.1 \\
\hline 110 & 1045.2 & 1037.6 & 1028.1 & 1017.7 & 1005.9 & 993.0 \\
\hline 120 & 1048.8 & 1041.1 & 1031.7 & 1021.2 & 1009.6 & 996.7 \\
\hline \multicolumn{7}{|c|}{$w_{1}=0.2000$} \\
\hline 0.1 & 1006.9 & 999.1 & 988.8 & 976.7 & 962.5 & 946.5 \\
\hline 0.5 & 1007.0 & 999.2 & 988.9 & 976.7 & 962.7 & 946.9 \\
\hline 1 & 1007.2 & 999.4 & 989.1 & 976.9 & 962.9 & 947.0 \\
\hline 2 & 1007.6 & 999.8 & 989.5 & 977.4 & 963.4 & 947.5 \\
\hline 5 & 1008.7 & 1001.0 & 990.7 & 978.6 & 964.7 & 949.0 \\
\hline 10 & 1010.7 & 1002.9 & 992.7 & 980.8 & 966.9 & 951.5 \\
\hline 15 & 1012.6 & 1004.8 & 994.7 & 982.9 & 969.1 & 953.8 \\
\hline 20 & 1014.5 & 1006.7 & 996.6 & 984.9 & 971.3 & 956.2 \\
\hline 30 & 1018.4 & 1010.5 & 1000.5 & 988.9 & 975.6 & 960.7 \\
\hline 40 & 1022.1 & 1014.3 & 1004.5 & 992.9 & 979.8 & 965.2 \\
\hline 50 & 1025.8 & 1017.9 & 1008.0 & 996.7 & 983.7 & 969.4 \\
\hline 60 & 1029.4 & 1021.5 & 1011.7 & 1000.6 & 987.7 & 973.6 \\
\hline 70 & 1033.0 & 1025.0 & 1015.2 & 1004.2 & 991.5 & 977.8 \\
\hline
\end{tabular}




\begin{tabular}{|c|c|c|c|c|c|c|}
\hline 80 & 1036.2 & 1028.5 & 1018.7 & 1007.7 & 995.4 & 981.6 \\
\hline 90 & 1039.7 & 1031.8 & 1022.1 & 1011.3 & 999.0 & 985.6 \\
\hline 100 & 1043.2 & 1035.1 & 1025.5 & 1014.7 & 1002.5 & 989.4 \\
\hline 110 & 1046.4 & 1038.4 & 1028.8 & 1018.2 & 1006.1 & 993.0 \\
\hline 120 & 1049.6 & 1041.8 & 1032.2 & 1021.6 & 1009.6 & 996.7 \\
\hline & \multicolumn{6}{|c|}{$w_{1}=0.3005$} \\
\hline 0.1 & 1012.3 & 1003.2 & 992.0 & 979.2 & 964.5 & 948.4 \\
\hline 0.5 & 1012.5 & 1003.4 & 992.3 & 979.4 & 964.8 & 948.8 \\
\hline 1 & 1012.7 & 1003.6 & 992.4 & 979.6 & 965.0 & 948.9 \\
\hline 2 & 1013.0 & 1004.0 & 992.8 & 980.0 & 965.5 & 949.4 \\
\hline 5 & 1014.1 & 1005.1 & 994.0 & 981.2 & 966.8 & 950.9 \\
\hline 10 & 1016.0 & 1007.0 & 996.0 & 983.3 & 969.0 & 953.3 \\
\hline 15 & 1017.7 & 1008.8 & 997.9 & 985.4 & 971.2 & 955.7 \\
\hline 20 & 1019.6 & 1010.6 & 999.7 & 987.4 & 973.4 & 958.1 \\
\hline 30 & 1023.2 & 1014.3 & 1003.5 & 991.4 & 977.6 & 962.6 \\
\hline 40 & 1026.6 & 1017.9 & 1007.3 & 995.2 & 981.7 & 967.0 \\
\hline 50 & 1030.0 & 1021.3 & 1010.8 & 999.0 & 985.6 & 971.1 \\
\hline 60 & 1033.5 & 1024.8 & 1014.4 & 1002.7 & 989.5 & 975.3 \\
\hline 70 & 1036.9 & 1028.2 & 1017.8 & 1006.2 & 993.3 & 979.5 \\
\hline 80 & 1040.0 & 1031.4 & 1021.1 & 1009.8 & 997.1 & 983.2 \\
\hline 90 & 1043.3 & 1034.5 & 1024.4 & 1013.1 & 1000.8 & 987.1 \\
\hline 100 & 1046.6 & 1037.9 & 1027.7 & 1016.6 & 1004.2 & 990.9 \\
\hline 110 & 1049.6 & 1041.0 & 1031.0 & 1020.0 & 1007.7 & 994.6 \\
\hline 120 & 1052.6 & 1044.1 & 1034.2 & 1023.3 & 1011.1 & 998.2 \\
\hline \multicolumn{7}{|c|}{$w_{1}=0.4000$} \\
\hline 0.1 & 1018.1 & 1007.5 & 995.3 & 981.9 & 966.6 & 950.0 \\
\hline 0.5 & 1018.1 & 1007.7 & 995.6 & 982.0 & 966.9 & 950.4 \\
\hline 1 & 1018.3 & 1007.9 & 995.8 & 982.2 & 967.1 & 950.6 \\
\hline
\end{tabular}




\begin{tabular}{rrrrrrr}
2 & 1018.6 & 1008.3 & 996.2 & 982.6 & 967.6 & 951.1 \\
5 & 1019.6 & 1009.3 & 997.3 & 983.9 & 968.9 & 952.5 \\
10 & 1021.4 & 1011.2 & 999.3 & 986.0 & 971.1 & 955.0 \\
15 & 1023.1 & 1012.9 & 1001.1 & 988.0 & 973.3 & 957.3 \\
20 & 1024.8 & 1014.7 & 1003.0 & 990.0 & 975.4 & 959.7 \\
30 & 1028.3 & 1018.2 & 1006.7 & 993.9 & 979.7 & 964.3 \\
40 & 1031.6 & 1021.7 & 1010.4 & 997.7 & 983.8 & 968.7 \\
50 & 1034.8 & 1025.1 & 1013.8 & 1001.4 & 987.6 & 972.8 \\
60 & 1038.1 & 1028.4 & 1017.3 & 1005.1 & 991.6 & 977.0 \\
70 & 1041.4 & 1031.7 & 1020.7 & 1008.6 & 995.2 & 981.1 \\
80 & 1044.3 & 1034.8 & 1024.0 & 1012.1 & 999.1 & 985.0 \\
90 & 1047.5 & 1037.8 & 1027.1 & 1015.3 & 1002.5 & 988.8 \\
100 & 1050.4 & 1041.1 & 1030.3 & 1018.7 & 1006.0 & 992.5 \\
110 & 1053.3 & 1044.0 & 1033.5 & 1022.1 & 1009.5 & 996.2 \\
120 & 1056.3 & 1047.0 & 1036.7 & 1025.2 & 1012.9 & 999.7 \\
\hline
\end{tabular}

${ }^{\mathrm{a}}$ Standard uncertainties $u$ are: $u(T)=0.01 \mathrm{~K} ; u_{\mathrm{r}}(p)=0.0001(\mathrm{kPa} / \mathrm{kPa}) ; u(w)=0.0001$ and $u(\rho)=0.35 \mathrm{~kg} \cdot \mathrm{m}^{-3}$

Density measurements show that densities of aqueous solutions of MDEA are always higher than the densities of aqueous solutions of MEA for the same conditions of temperature, pressure and composition. The density of these mixtures increases when the weight fraction of the amine is higher and this effect is greater for the solutions with MDEA. Thus, the density differences between MDEA and MEA solutions are higher when the weight fraction of the amine increases. As is expected, density increases when pressure increases or temperature decreases, and these effects are similar for both amine solutions.

The experimental values were correlated using a modified Tammann-Tait equation (equation (3)) for each composition: 


$$
\rho(T, p)=\frac{A_{0}+A_{1} T+A_{2} T^{2}}{1-C \ln \left(\frac{B_{0}+B_{1} T+B_{2} T^{2}+p}{B_{0}+B_{1} T+B_{2} T^{2}+0.1 M P a}\right)}
$$

These results are given in table 7 which contains the fitting parameters and the standard deviation. As can be seen, the experimental results correlate quite well using this equation (3). Although better results are obtained for the mixtures with MDEA, standard deviations are lower than density uncertainties for both amines. 
Table 7. Fitting parameters of equation (3) and standard deviations $\sigma$ for the density measurements

\begin{tabular}{|c|c|c|c|c|}
\hline $\operatorname{MDEA}(1)+\mathrm{H}_{2} \mathrm{O}(2)$ & $w_{1}=0.1$ & $w_{1}=0.2$ & $w_{1}=0.3$ & $w_{1}=0.4$ \\
\hline $\mathrm{A}_{0} / \mathrm{kg} \cdot \mathrm{m}^{-3}$ & 854.7268 & 897.2473 & 955.0871 & 1017.113 \\
\hline $\mathrm{A}_{1} / \mathrm{kg} \cdot \mathrm{m}^{-3} \cdot \mathrm{K}^{-1}$ & 1.35021 & 1.17809 & 0.92985 & 0.65632 \\
\hline $\mathrm{A}_{2} / \mathrm{kg} \cdot \mathrm{m}^{-3} \cdot \mathrm{K}^{-2}$ & -0.002830 & -0.002630 & -0.002337 & -0.002012 \\
\hline $\mathrm{B}_{0} / \mathrm{MPa}$ & -675.7028 & -128.6144 & 36.29279 & 631.3078 \\
\hline $\mathrm{B}_{1} / \mathrm{MPa} \cdot \mathrm{K}^{-1}$ & 6.82909 & 3.15987 & 2.14289 & -0.49223 \\
\hline $\mathrm{B}_{2} / \mathrm{MPa} \cdot \mathrm{K}^{-2}$ & -0.011016 & -0.005719 & -0.004442 & -0.5996 \\
\hline $\mathrm{C}$ & 0.157010 & 0.121019 & 0.104427 & 0.108548 \\
\hline$\sigma / \mathrm{kg} \cdot \mathrm{m}^{-3}$ & 0.029 & 0.018 & 0.0046 & 0.026 \\
\hline $\operatorname{MEA}(1)+\mathrm{H}_{2} \mathrm{O}(2)$ & $w_{1}=0.1$ & $w_{1}=0.2$ & $w_{1}=0.3$ & $w_{1}=0.4$ \\
\hline $\mathrm{A}_{0} / \mathrm{kg} \cdot \mathrm{m}^{-3}$ & 850.8313 & 893.1019 & 948.8575 & 998.3203 \\
\hline $\mathrm{A}_{1} / \mathrm{kg} \cdot \mathrm{m}^{-3} \cdot \mathrm{K}^{-1}$ & 1.33316 & 1.12950 & 0.85601 & 0.62575 \\
\hline $\mathrm{A}_{2} / \mathrm{kg} \cdot \mathrm{m}^{-3} \cdot \mathrm{K}^{-2}$ & -0.002781 & -0.002528 & -0.002181 & -0.001904 \\
\hline $\mathrm{B}_{0} / \mathrm{MPa}$ & -558.9608 & -258.7132 & 73.8801 & 323.1854 \\
\hline $\mathrm{B}_{1} / \mathrm{MPa} \cdot \mathrm{K}^{-1}$ & 5.50608 & 3.96741 & 2.15210 & 0.78340 \\
\hline $\mathrm{B}_{2} / \mathrm{MPa} \cdot \mathrm{K}^{-2}$ & -0.008807 & -0.006845 & -0.004437 & -0.002664 \\
\hline $\mathrm{C}$ & 0.130217 & 0.126603 & 0.120879 & 0.108548 \\
\hline$\sigma / \mathrm{kg} \cdot \mathrm{m}^{-3}$ & 0.171 & 0.134 & 0.113 & 0.120 \\
\hline
\end{tabular}


We have mainly found density data at atmospheric pressure in literature for comparison. For aqueous solutions of MDEA, Rinker et al. [19] measured the same compositions at $T=$ (333.15 to 373.15$) \mathrm{K}$, with the average absolute deviation between our data and the twelve points compared being $0.46 \mathrm{~kg} \cdot \mathrm{m}^{-3}$. This value is consistent with the uncertainty of our measurements as occurs with other authors [20-25], the relative absolute deviations ranging from $0.02 \%$ to $0.08 \%$. Zúñiga-Moreno et al. [26] published density data for this mixture up to $20 \mathrm{MPa}$, the deviations for the composition $\mathrm{w}_{1}=0.3$ are consistent with the uncertainty but this is not true for $\mathrm{w}_{1}=0.2$; it could be attributed to a slight difference in the composition. The relative deviations are shown graphically in Figure 3.

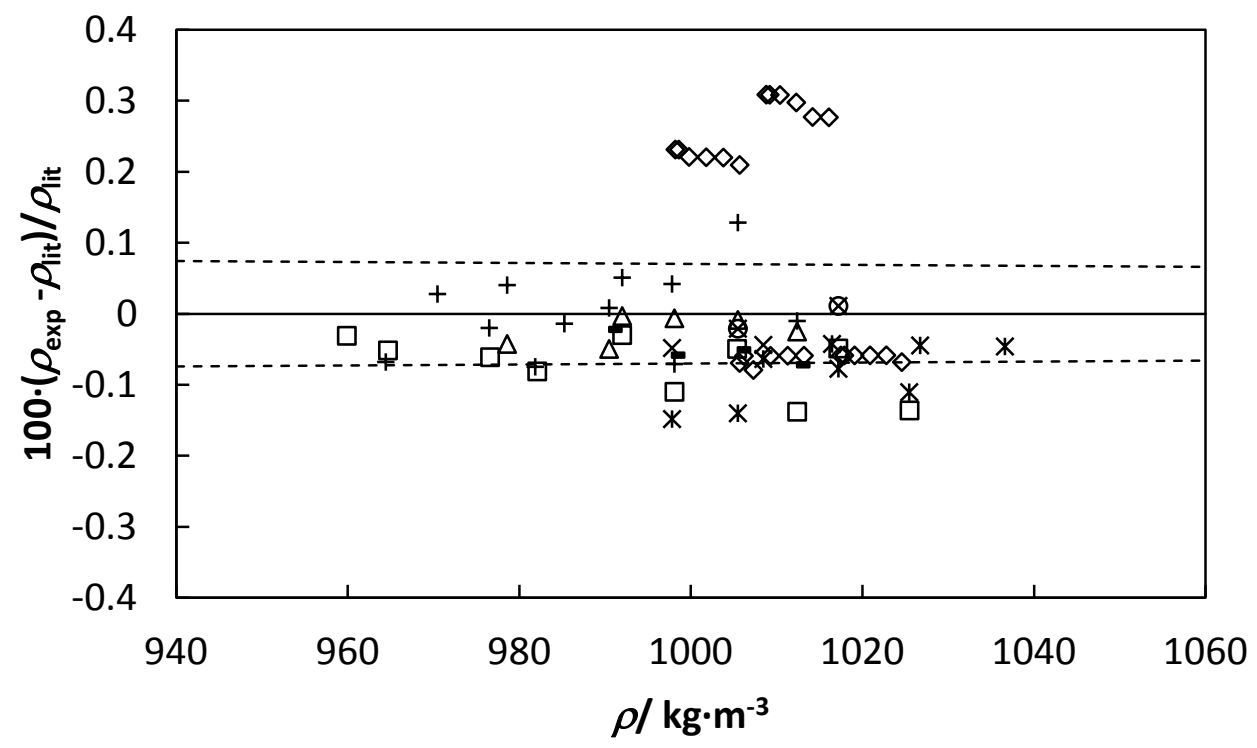

Figure 3. Relative deviation (\%) of the experimental data of MDEA $+\mathrm{H}_{2} \mathrm{O}$ density from the literature as a function of density: + Rinker et al. [19]; * Al-Ghawas et al. [20]; ○ Li et al. [21]; $\times$ Li et al. [22]; $\Delta$ Bernal-García et al. [23]; $\square$ Han et al. [24]; - Pouryousefi et al. [25] and $\diamond$ Zúñiga-Moreno et al. [26]. Dotted lines represent the relative expanded uncertainty of our measurements. 
As regards MEA solutions, the relative absolute deviations, with different literature data, were $0.02 \%$ [25], with the same range of compositions and three temperatures at $p=0.1 \mathrm{MPa}$ (twelve points in common), $0.14 \%$ [27] (only two points for comparison) $0.04 \%$ [28] (one point), $0.01 \%$ (four points in common) [29] and 0.06\% [30] (ten points for comparison). Figure 4 shows these relative deviations.

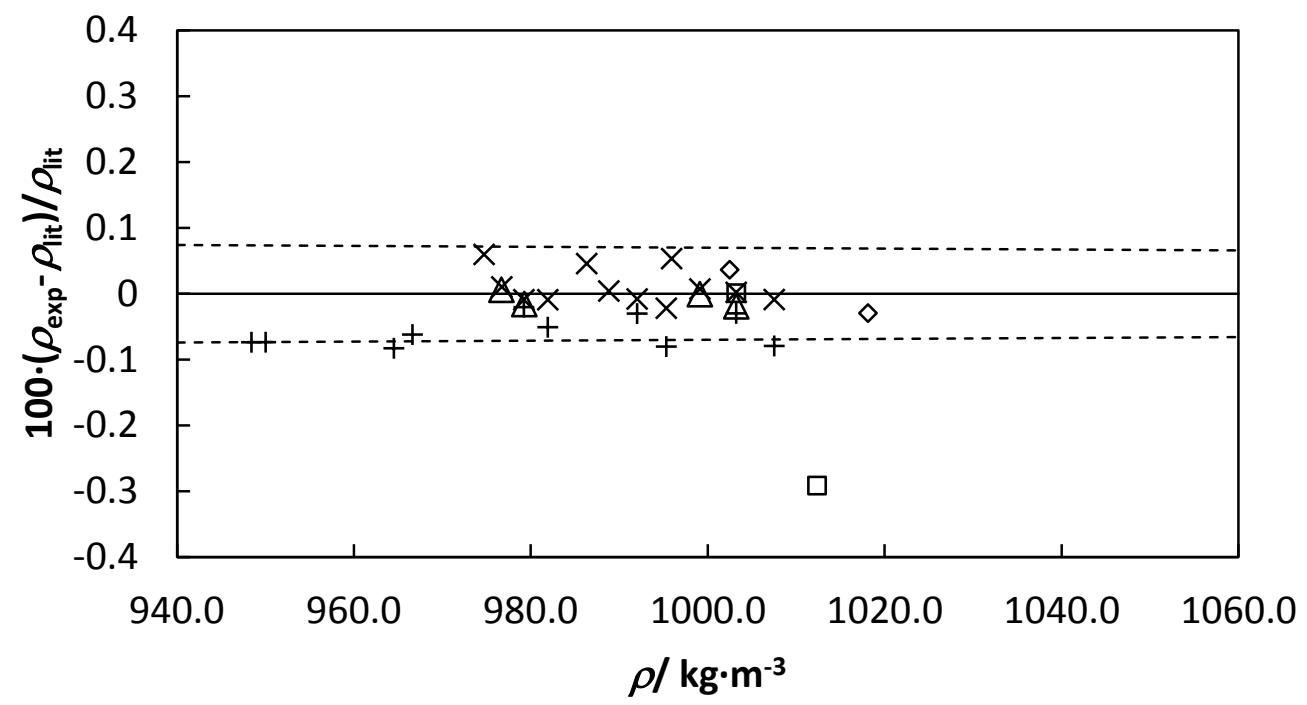

Figure 4. Relative deviation (\%) of the experimental data of MEA $+\mathrm{H}_{2} \mathrm{O}$ density from the literature as a function of density: $\times$ Pouryousefi et al. [25] ; $\square$ Paul et al. [27]; $\diamond$ Tseng et al. [28]; $\Delta$ Amundsen et al. [29] and + Han et al. [30]. Dotted lines represent the relative expanded uncertainty of our measurements.

Viscosity measurements of aqueous solutions of methyldiethanolamine (MDEA) and monoethanolamina (MEA) were performed for different amine mass fractions: $0.1,0.2,0.3$ and 0.4 , at $p=(0.1$ to 120$) \mathrm{MPa}$ and four temperatures from 293.15 to $353.15 \mathrm{~K}$ using the falling body viscometer. Experimental data are presented in table 8 for MDEA and table 9 for MEA. 
It is important to remember the limits of the calibration used $\eta=(0.630$ to 4.929$) \mathrm{mPa} \cdot \mathrm{s}$. This is why viscosities of $10 \%$ w MDEA $+\mathrm{H}_{2} \mathrm{O}$ at $T=353.15 \mathrm{~K}$, of $40 \% \mathrm{w}$ MDEA $+\mathrm{H}_{2} \mathrm{O}$ at $T=$ 293.15 K, viscosities of $10 \%$ w MEA $+\mathrm{H}_{2} \mathrm{O}$ at $T=353.15 \mathrm{~K}$ or of $20 \% \mathrm{w}$ MEA $+\mathrm{H}_{2} \mathrm{O}$ at $T=$ 353.15 K (up to $40 \mathrm{MPa}$ ) are not given, since said viscosities were outside the calibration range and could not thus be measured using this calibration. However, there are certain viscosity values which are slightly below or above these limits imposed by calibration with $n$ dodecane which might be permissible considering their uncertainties.

Table 8. Experimental dynamic viscosities $\eta$, for the MDEA (1) $+\mathrm{H}_{2} \mathrm{O}$ (2) system at different conditions of temperature, $T$, pressure, $p$, and mass fraction, $w_{1}{ }^{\text {a }}$

\begin{tabular}{cccccccccc}
\hline$T / \mathrm{K}$ & $p / \mathrm{MPa}$ & $w_{1}$ & $\eta / \mathrm{mPa} \cdot \mathrm{s}$ & $w_{1}$ & $\eta / \mathrm{mPa} \cdot \mathrm{s}$ & $w_{1}$ & $\eta / \mathrm{mPa} \cdot \mathrm{s}$ & $w_{1}$ & $\eta / \mathrm{mPa} \cdot \mathrm{s}$ \\
\hline 293.15 & 0.1 & 0.1000 & 1.476 & 0.2002 & 2.350 & 0.3000 & 3.999 & & \\
293.15 & 5 & 0.1000 & 1.475 & 0.2002 & 2.353 & 0.3000 & 4.018 & & \\
293.15 & 10 & 0.1000 & 1.475 & 0.2002 & 2.357 & 0.3000 & 4.038 & & \\
293.15 & 20 & 0.1000 & 1.471 & 0.2002 & 2.364 & 0.3000 & 4.077 & & \\
293.15 & 30 & 0.1000 & 1.472 & 0.2002 & 2.372 & 0.3000 & 4.114 & & \\
293.15 & 40 & 0.1000 & 1.470 & 0.2002 & 2.380 & 0.3000 & 4.153 & & \\
293.15 & 60 & 0.1000 & 1.474 & 0.2002 & 2.399 & 0.3000 & 4.231 & & \\
293.15 & 80 & 0.1000 & 1.477 & 0.2002 & 2.419 & 0.3000 & 4.311 & & \\
293.15 & 100 & 0.1000 & 1.485 & 0.2002 & 2.437 & 0.3000 & 4.390 & & \\
293.15 & 120 & 0.1000 & 1.492 & 0.2002 & 2.464 & 0.3000 & 4.478 & & \\
313.15 & 0.1 & 0.1000 & 0.912 & 0.2002 & 1.345 & 0.3000 & 2.095 & 0.4000 & 3.238 \\
313.15 & 5 & 0.1000 & 0.914 & 0.2002 & 1.347 & 0.3000 & 2.106 & 0.4000 & 3.270 \\
313.15 & 10 & 0.1000 & 0.915 & 0.2002 & 1.349 & 0.3000 & 2.118 & 0.4000 & 3.302
\end{tabular}




\begin{tabular}{|c|c|c|c|c|c|c|c|c|c|}
\hline 313.15 & 20 & 0.1000 & 0.920 & 0.2002 & 1.358 & 0.3000 & 2.144 & 0.4000 & 3.365 \\
\hline 313.15 & 30 & 0.1000 & 0.923 & 0.2002 & 1.367 & 0.3000 & 2.168 & 0.4000 & 3.428 \\
\hline 313.15 & 40 & 0.1000 & 0.927 & 0.2002 & 1.377 & 0.3000 & 2.192 & 0.4000 & 3.491 \\
\hline 313.15 & 60 & 0.1000 & 0.936 & 0.2002 & 1.398 & 0.3000 & 2.244 & 0.4000 & 3.618 \\
\hline 313.15 & 80 & 0.1000 & 0.945 & 0.2002 & 1.418 & 0.3000 & 2.296 & 0.4000 & 3.740 \\
\hline 313.15 & 100 & 0.1000 & 0.955 & 0.2002 & 1.445 & 0.3000 & 2.356 & 0.4000 & 3.866 \\
\hline 313.15 & 120 & 0.1000 & 0.964 & 0.2002 & 1.466 & 0.3000 & 2.412 & 0.4000 & 3.996 \\
\hline 333.15 & 0.1 & 0.1000 & 0.624 & 0.2002 & 0.872 & 0.3000 & 1.271 & 0.4000 & 1.843 \\
\hline 333.15 & 5 & 0.1000 & 0.627 & 0.2002 & 0.876 & 0.3000 & 1.278 & 0.4000 & 1.861 \\
\hline 333.15 & 10 & 0.1000 & 0.630 & 0.2002 & 0.881 & 0.3000 & 1.286 & 0.4000 & 1.880 \\
\hline 333.15 & 20 & 0.1000 & 0.634 & 0.2002 & 0.890 & 0.3000 & 1.302 & 0.4000 & 1.921 \\
\hline 333.15 & 30 & 0.1000 & 0.638 & 0.2002 & 0.898 & 0.3000 & 1.316 & 0.4000 & 1.956 \\
\hline 333.15 & 40 & 0.1000 & 0.643 & 0.2002 & 0.907 & 0.3000 & 1.332 & 0.4000 & 1.992 \\
\hline 333.15 & 60 & 0.1000 & 0.652 & 0.2002 & 0.924 & 0.3000 & 1.367 & 0.4000 & 2.063 \\
\hline 333.15 & 80 & 0.1000 & 0.661 & 0.2002 & 0.942 & 0.3000 & 1.400 & 0.4000 & 2.134 \\
\hline 333.15 & 100 & 0.1000 & 0.672 & 0.2002 & 0.967 & 0.3000 & 1.435 & 0.4000 & 2.206 \\
\hline 333.15 & 120 & 0.1000 & 0.680 & 0.2002 & 0.982 & 0.3000 & 1.468 & 0.4000 & 2.280 \\
\hline 353.15 & 0.1 & & & 0.2002 & 0.617 & 0.3000 & 0.854 & 0.4000 & 1.181 \\
\hline 353.15 & 5 & & & 0.2002 & 0.620 & 0.3000 & 0.862 & 0.4000 & 1.193 \\
\hline 353.15 & 10 & & & 0.2002 & 0.625 & 0.3000 & 0.869 & 0.4000 & 1.204 \\
\hline 353.15 & 20 & & & 0.2002 & 0.633 & 0.3000 & 0.881 & 0.4000 & 1.229 \\
\hline 353.15 & 30 & & & 0.2002 & 0.640 & 0.3000 & 0.894 & 0.4000 & 1.253 \\
\hline 353.15 & 40 & & & 0.2002 & 0.647 & 0.3000 & 0.907 & 0.4000 & 1.276 \\
\hline 353.15 & 60 & & & 0.2002 & 0.661 & 0.3000 & 0.932 & 0.4000 & 1.322 \\
\hline 353.15 & 80 & & & 0.2002 & 0.675 & 0.3000 & 0.959 & 0.4000 & 1.368 \\
\hline
\end{tabular}


353.15100

$\begin{array}{llllll}0.2002 & 0.691 & 0.3000 & 0.997 & 0.4000 & 1.419\end{array}$

$353.15 \quad 120$

0.2002

0.705

0.3000

$1.018 \quad 0.4000$

1.467

${ }^{\mathrm{a}}$ Standard uncertainties $u$ are: $u(T)=0.01 \mathrm{~K} ; u_{\mathrm{r}}(p)=0.0001(\mathrm{kPa} / \mathrm{kPa}) ; u(w)=0.0001 ; u_{\mathrm{r}}(\eta)=$ $0.015(\mathrm{mPa} \cdot \mathrm{s} / \mathrm{mPa} \cdot \mathrm{s})$

Table 9. Experimental dynamic viscosities $\eta$, for the MEA (1) $+\mathrm{H}_{2} \mathrm{O}$ (2) system at different conditions of temperature, $T$, pressure, $p$, and mass fraction, $w_{1}{ }^{\mathrm{a}}$

\begin{tabular}{|c|c|c|c|c|c|c|c|c|c|}
\hline$T / \mathrm{K}$ & $p / \mathrm{MPa}$ & $w_{1}$ & $\eta / \mathrm{mPa} \cdot \mathrm{s}$ & $w_{1}$ & $\eta / \mathrm{mPa} \cdot \mathrm{s}$ & $w_{1}$ & $\eta / \mathrm{mPa} \cdot \mathrm{s}$ & $w_{1}$ & $\eta / \mathrm{mPa} \cdot \mathrm{s}$ \\
\hline 293.15 & 0.1 & 0.1001 & 1.435 & 0.2000 & 1.968 & 0.3005 & 2.913 & 0.4001 & 4.315 \\
\hline 293.15 & 5 & 0.1001 & 1.435 & 0.2000 & 1.970 & 0.3005 & 2.921 & 0.4001 & 4.340 \\
\hline 293.15 & 10 & 0.1001 & 1.434 & 0.2000 & 1.969 & 0.3005 & 2.930 & 0.4001 & 4.368 \\
\hline 293.15 & 20 & 0.1001 & 1.430 & 0.2000 & 1.973 & 0.3005 & 2.952 & 0.4001 & 4.426 \\
\hline 293.15 & 30 & 0.1001 & 1.430 & 0.2000 & 1.977 & 0.3005 & 2.972 & 0.4001 & 4.475 \\
\hline 293.15 & 40 & 0.1001 & 1.429 & 0.2000 & 1.983 & 0.3005 & 2.990 & 0.4001 & 4.528 \\
\hline 293.15 & 60 & 0.1001 & 1.431 & 0.2000 & 1.994 & 0.3005 & 3.032 & 0.4001 & 4.628 \\
\hline 293.15 & 80 & 0.1001 & 1.434 & 0.2000 & 2.009 & 0.3005 & 3.076 & 0.4001 & 4.739 \\
\hline 293.15 & 100 & 0.1001 & 1.432 & 0.2000 & 2.026 & 0.3005 & 3.120 & 0.4001 & 4.845 \\
\hline 293.15 & 120 & 0.1001 & 1.443 & 0.2000 & 2.046 & 0.3005 & 3.162 & 0.4001 & 4.954 \\
\hline 313.15 & 0.1 & 0.1001 & 0.891 & 0.2000 & 1.173 & 0.3005 & 1.638 & 0.4001 & 2.284 \\
\hline 313.15 & 5 & 0.1001 & 0.892 & 0.2000 & 1.178 & 0.3005 & 1.642 & 0.4001 & 2.306 \\
\hline 313.15 & 10 & 0.1001 & 0.892 & 0.2000 & 1.182 & 0.3005 & 1.649 & 0.4001 & 2.319 \\
\hline 313.15 & 20 & 0.1001 & 0.896 & 0.2000 & 1.190 & 0.3005 & 1.668 & 0.4001 & 2.352 \\
\hline 313.15 & 30 & 0.1001 & 0.899 & 0.2000 & 1.197 & 0.3005 & 1.686 & 0.4001 & 2.384 \\
\hline 313.15 & 40 & 0.1001 & 0.902 & 0.2000 & 1.205 & 0.3005 & 1.702 & 0.4001 & 2.416 \\
\hline 313.15 & 60 & 0.1001 & 0.910 & 0.2000 & 1.219 & 0.3005 & 1.735 & 0.4001 & 2.479 \\
\hline
\end{tabular}




\begin{tabular}{|c|c|c|c|c|c|c|c|c|c|}
\hline 313.15 & 80 & 0.1001 & 0.918 & 0.2000 & 1.232 & 0.3005 & 1.770 & 0.4001 & 2.544 \\
\hline 313.15 & 100 & 0.1001 & 0.927 & 0.2000 & 1.249 & 0.3005 & 1.804 & 0.4001 & 2.609 \\
\hline 313.15 & 120 & 0.1001 & 0.936 & 0.2000 & 1.263 & 0.3005 & 1.846 & 0.4001 & 2.675 \\
\hline 333.15 & 0.1 & 0.1001 & 0.615 & 0.2000 & 0.780 & 0.3005 & 1.061 & 0.4001 & 1.389 \\
\hline 333.15 & 5 & 0.1001 & 0.614 & 0.2000 & 0.788 & 0.3005 & 1.067 & 0.4001 & 1.401 \\
\hline 333.15 & 10 & 0.1001 & 0.617 & 0.2000 & 0.792 & 0.3005 & 1.075 & 0.4001 & 1.410 \\
\hline 333.15 & 20 & 0.1001 & 0.622 & 0.2000 & 0.799 & 0.3005 & 1.089 & 0.4001 & 1.434 \\
\hline 333.15 & 30 & 0.1001 & 0.625 & 0.2000 & 0.807 & 0.3005 & 1.103 & 0.4001 & 1.453 \\
\hline 333.15 & 40 & 0.1001 & 0.630 & 0.2000 & 0.815 & 0.3005 & 1.115 & 0.4001 & 1.475 \\
\hline 333.15 & 60 & 0.1001 & 0.639 & 0.2000 & 0.829 & 0.3005 & 1.140 & 0.4001 & 1.518 \\
\hline 333.15 & 80 & 0.1001 & 0.646 & 0.2000 & 0.844 & 0.3005 & 1.165 & 0.4001 & 1.559 \\
\hline 333.15 & 100 & 0.1001 & 0.655 & 0.2000 & 0.859 & 0.3005 & 1.189 & 0.4001 & 1.600 \\
\hline 333.15 & 120 & 0.1001 & 0.663 & 0.2000 & 0.874 & 0.3005 & 1.211 & 0.4001 & 1.645 \\
\hline 353.15 & 0.1 & 0.1001 & & 0.2000 & & 0.3005 & 0.746 & 0.4001 & 0.942 \\
\hline 353.15 & 5 & 0.1001 & & 0.2000 & & 0.3005 & 0.748 & 0.4001 & 0.944 \\
\hline 353.15 & 10 & 0.1001 & & 0.2000 & & 0.3005 & 0.755 & 0.4001 & 0.957 \\
\hline 353.15 & 20 & 0.1001 & & 0.2000 & & 0.3005 & 0.768 & 0.4001 & 0.975 \\
\hline 353.15 & 30 & 0.1001 & & 0.2000 & & 0.3005 & 0.777 & 0.4001 & 0.989 \\
\hline 353.15 & 40 & 0.1001 & & 0.2000 & & 0.3005 & 0.787 & 0.4001 & 1.006 \\
\hline 353.15 & 60 & 0.1001 & & 0.2000 & 0.607 & 0.3005 & 0.807 & 0.4001 & 1.039 \\
\hline 353.15 & 80 & 0.1001 & & 0.2000 & 0.619 & 0.3005 & 0.828 & 0.4001 & 1.068 \\
\hline 353.15 & 100 & 0.1001 & & 0.2000 & 0.632 & 0.3005 & 0.847 & 0.4001 & 1.097 \\
\hline 353.15 & 120 & 0.1001 & & 0.2000 & 0.644 & 0.3005 & 0.861 & 0.4001 & 1.128 \\
\hline
\end{tabular}

${ }^{\mathrm{a}}$ Standard uncertainties $u$ are: $u(T)=0.01 \mathrm{~K} ; u_{\mathrm{r}}(p)=0.0001(\mathrm{kPa} / \mathrm{kPa}) ; u(w)=0.0001 ; u_{\mathrm{r}}(\eta)=$ $0.015(\mathrm{mPa} \cdot \mathrm{s} / \mathrm{mPa} \cdot \mathrm{s})$ 
Viscosities of aqueous solutions of MDEA are always higher than viscosities of aqueous solutions of MEA under the same conditions of temperature, pressure and composition, as was also observed for densities. Viscosities of these mixtures increase at greater amine weight fractions and this effect is slightly higher for solutions with MDEA. Viscosity increases when pressure increases or temperature decreases, although the effect of temperature is much greater. These effects are similar for both amine solutions. Moreover, the increase in viscosity for higher amine weight fractions is more significant at lower temperatures.

Experimental viscosities were correlated using a modified VFT equation proposed by Comuñas et al. [31]:

$\eta(T, p)=A \exp \left\lfloor\frac{B}{T-C}\right\rfloor \exp \left\lfloor D \ln \left(\frac{p+E(T)}{0.1+E(T)}\right)\right\rfloor$

where

$E(T)=E_{0}+E_{1} T+E_{2} T^{2}$

The results of the correlation, which include the fitting parameters and the standard deviations, are summarized in table 10. The standard deviations obtained from the fittings are lower than the uncertainties of the experimental measurements and thus the correlation model is suitable for describing the viscosity behaviour of this kind of mixtures.

Table 10. Fitting parameters of equation (4) and standard deviation for the viscosity measurements.

\begin{tabular}{lcccc}
\hline $\mathrm{MDEA}(1)+\mathrm{H}_{2} \mathrm{O}(2)$ & $w_{1}=0.1$ & $w_{1}=0.2$ & $w_{1}=0.3$ & $w_{1}=0.4$ \\
\hline $\mathrm{A} / \mathrm{mPa} \cdot \mathrm{s}$ & 0.0015 & 0.0014 & 0.0013 & 0.0018 \\
$\mathrm{~B} / \mathrm{K}$ & 1901.9 & 1903.5 & 1904.9 & 1905.1 \\
$\mathrm{C} / \mathrm{K}$ & 17.572 & 37.900 & 57.019 & 59.773 \\
$\mathrm{D}$ & 1.0238 & 1.3329 & 4.9634 & 0.9208
\end{tabular}




\begin{tabular}{lllll}
$\mathrm{E}_{0} / \mathrm{MPa}$ & 2.3741 & 1.4751 & 1.4549 & -0.1803 \\
$\mathrm{E}_{1} / \mathrm{MPa} \cdot \mathrm{K}^{-1}$ & 214.85 & 76.776 & 72.212 & 3.5386 \\
$\mathrm{E}_{2} / \mathrm{MPa} \cdot \mathrm{K}^{-2}$ & -0.6354 & -0.2212 & -0.1853 & -0.0065 \\
$\sigma / \mathrm{mPa} \cdot \mathrm{s}$ & 0.0080 & 0.0122 & 0.0213 & 0.0190 \\
\hline $\mathrm{MEA}(1)+\mathrm{H}_{2} \mathrm{O}(2)$ & $w_{1}=0.1$ & $w_{1}=0.2$ & $w_{1}=0.3$ & $w_{1}=0.4$ \\
\hline $\mathrm{A} / \mathrm{mPa} \cdot \mathrm{s}$ & 0.0015 & 0.0015 & 0.0015 & 0.0014 \\
$\mathrm{~B} / \mathrm{K}$ & 1901.8 & 1902.7 & 1903.7 & 1904.8 \\
$\mathrm{C} / \mathrm{K}$ & 15.502 & 27.922 & 40.915 & 55.404 \\
$\mathrm{D}$ & 0.9940 & 0.9488 & 3.0065 & 0.8551 \\
$\mathrm{E}_{0} / \mathrm{MPa}$ & 3.0884 & 1.4267 & 1.4864 & -0.7075 \\
$\mathrm{E}_{1} / \mathrm{MPa} \cdot \mathrm{K}^{-1}$ & 326.09 & 69.048 & 79.220 & 8.8161 \\
$\mathrm{E}_{2} / \mathrm{MPa} \cdot \mathrm{K}^{-2}$ & -0.9687 & -0.2005 & -0.2188 & -0.0220 \\
$\sigma / \mathrm{mPa} \cdot \mathrm{s}$ & 0.0078 & 0.0086 & 0.0205 & 0.0219 \\
\hline
\end{tabular}

In order to check the reliability of viscosity data, a comparison was carried out at atmospheric pressure (due to the lack of these values at high pressures) between viscosities obtained with our falling body viscometer and the ones obtained using a Stabinger SVM 3000 viscometer available in our laboratory (table 11).

Table 11. Viscosity comparison between falling body viscometer $(\mathrm{FB})^{\mathrm{a}}$ at $p=0.1 \mathrm{MPa}$ and Stabinger SVM 3000 viscometer $(\mathrm{SVM})^{\mathrm{b}}$ at $p=0.093 \mathrm{MPa}$ (atmospheric pressure) for the mixtures studied.

\begin{tabular}{cccccccc}
\hline & \multicolumn{3}{c}{ MDEA (1) $+\mathrm{H}_{2} \mathrm{O}(2)$} & \multicolumn{3}{c}{$\operatorname{MEA}(1)+\mathrm{H}_{2} \mathrm{O}(2)$} \\
\cline { 3 - 7 }$w_{1}$ & $T / \mathrm{K}$ & $\eta_{\mathrm{FB}} / \mathrm{mPa} \cdot \mathrm{s}$ & $\eta_{\mathrm{SVM}} / \mathrm{mPa} \cdot \mathrm{s}$ & $\%$ deviation & $\eta_{\mathrm{FB}} / \mathrm{mPa} \cdot \mathrm{s}$ & $\eta_{\mathrm{SVM}} / \mathrm{mPa} \cdot \mathrm{s}$ & $\%$ deviation \\
\hline 0.1 & 293.15 & 1.476 & 1.463 & 0.9 & 1.435 & 1.409 & 1.8 \\
0.1 & 313.15 & 0.912 & 0.9079 & 0.4 & 0.891 & 0.8932 & -0.3
\end{tabular}




\begin{tabular}{llllllll}
0.1 & 333.15 & 0.624 & 0.6344 & -1.6 & 0.615 & 0.6263 & -1.8 \\
0.2 & 293.15 & 2.350 & 2.293 & 2.4 & 1.968 & 1.982 & -0.7 \\
0.2 & 313.15 & 1.345 & 1.342 & 0.2 & 1.173 & 1.198 & -2.2 \\
0.2 & 333.15 & 0.872 & 0.8719 & 0.0 & 0.780 & 0.7832 & -0.4 \\
0.2 & 353.15 & 0.617 & 0.6205 & -0.6 & & & -1.9 \\
0.3 & 293.15 & 3.999 & 3.942 & 1.4 & 2.913 & 2.967 & -2.2 \\
0.3 & 313.15 & 2.095 & 2.094 & 0.0 & 1.638 & 1.675 & -2.1 \\
0.3 & 333.15 & 1.271 & 1.276 & -0.4 & 1.061 & 1.084 & -2.4 \\
0.3 & 353.15 & 0.854 & 0.8505 & 0.4 & 0.746 & 0.7640 & -0.3 \\
0.4 & 293.15 & & & & 4.315 & 4.326 & 0.0 \\
0.4 & 313.15 & 3.238 & 3.145 & 2.9 & 2.284 & 2.284 & -1.2 \\
0.4 & 333.15 & 1.843 & 1.820 & 1.2 & 1.389 & 1.405 & 0.9518 \\
0.4 & 353.15 & 1.181 & 1.186 & -0.4 & 0.942 & & \\
\hline
\end{tabular}

${ }^{\mathrm{a}}$ Standard uncertainties $u$ are: $u(T)=0.01 \mathrm{~K} ; u_{\mathrm{r}}(p)=0.0001(\mathrm{kPa} / \mathrm{kPa}) ; u(w)=0.0001 ; u_{\mathrm{r}}(\eta)=$ $0.015(\mathrm{mPa} \cdot \mathrm{s} / \mathrm{mPa} \cdot \mathrm{s})$

${ }^{\mathrm{b}}$ Standard uncertainties $u$ are: $u(T)=0.02 \mathrm{~K} ; u_{\mathrm{r}}(p)=0.005(\mathrm{kPa} / \mathrm{kPa}) ; u(w)=0.0001 ; u_{\mathrm{r}}(\eta)=$ $0.005(\mathrm{mPa} \cdot \mathrm{s} / \mathrm{mPa} \cdot \mathrm{s})$

As regards the compatibility of the results obtained using the falling body viscometer and the Stabinger viscometer, the maximum deviation is $2.9 \%$ which corresponds to the mixture 40 \% w MDEA at $T=313.15 \mathrm{~K}(3.2382 \mathrm{mPa} \cdot \mathrm{s}$ given by $\mathrm{FB}$ vs $3.1448 \mathrm{mPa} \cdot \mathrm{s}$ given by $\mathrm{SVM})$. This deviation is acceptable in light of the uncertainty values given for the falling body equipment combined with the expanded uncertainty of $\pm 1 \%$ for SVM 3000 .

After checking the compatibility of both viscometers, the values obtained with the falling body equipment will also be compared with literature values [19,20,22,23,27,32-35], at atmospheric pressure. A summary of the literature used in figure 5 is given in table 12 . 
Table 12. Literature data used to compare of the experimental viscosities measured in this work.

\begin{tabular}{|c|c|c|}
\hline Literature & systems & conditions \\
\hline \multirow{4}{*}{ Rinker et al. [19] } & \multirow{4}{*}{ MDEA (1) + $\mathrm{H}_{2} \mathrm{O}(2)$} & $w_{1}=0.1$ \\
\hline & & $\mathrm{T}=333.15 \mathrm{~K}$ \\
\hline & & $w_{1}=0.2 ; 0.3 ; 0.4$ \\
\hline & & $T=(333.15-353.15) \mathrm{K}$ \\
\hline \multirow{4}{*}{ Al-Ghawas et al. [20] } & \multirow{4}{*}{$\operatorname{MDEA}(1)+\mathrm{H}_{2} \mathrm{O}(2)$} & $w_{1}=0.1 ; 0.2 ; 0.3$ \\
\hline & & $T=(293.15-333.15) \mathrm{K}$ \\
\hline & & $w_{1}=0.4$ \\
\hline & & $T=313.15 \mathrm{~K}$ \\
\hline \multirow{6}{*}{ Li et al. [22] } & \multirow{4}{*}{$\operatorname{MDEA}(1)+\mathrm{H}_{2} \mathrm{O}(2)$} & $w_{1}=0.2 ; 0.3$ \\
\hline & & $T=(313.15-333.15) \mathrm{K}$ \\
\hline & & $w_{1}=0.4$ \\
\hline & & $T=313.15 \mathrm{~K}$ \\
\hline & \multirow{2}{*}{$\operatorname{MEA}(1)+\mathrm{H}_{2} \mathrm{O}(2)$} & $w_{1}=0.2 ; 0.3$ \\
\hline & & $T=(313.15-333.15) \mathrm{K}$ \\
\hline \multirow{2}{*}{ Paul et al. [27] } & \multirow{2}{*}{$\operatorname{MDEA}(1)+\mathrm{H}_{2} \mathrm{O}(2)$} & $w_{1}=0.1 ; 0.2 ; 0.3$ \\
\hline & & $T=(313.15-333.15) \mathrm{K}$ \\
\hline \multirow{4}{*}{ Arachchige et al. [32] } & \multirow{4}{*}{$\begin{array}{c}\operatorname{MDEA}(1)+\mathrm{H}_{2} \mathrm{O}(2) \\
\operatorname{MEA}(1)+\mathrm{H}_{2} \mathrm{O}(2)\end{array}$} & $w_{1}=0.1 ; 0.2$ \\
\hline & & $T=(293.15-333.15) \mathrm{K}$ \\
\hline & & $w_{1}=0.3 ; 0.4$ \\
\hline & & $T=(293.15-353.15) \mathrm{K}$ \\
\hline \multirow{2}{*}{ Teng et al. [33] } & \multirow{2}{*}{$\operatorname{MDEA}(1)+\mathrm{H}_{2} \mathrm{O}(2)$} & $w_{1}=0.1 ; 0.2 ; 0.3 ; 0.4$ \\
\hline & & $T=(313.15-353.15) \mathrm{K}$ \\
\hline \multirow{2}{*}{ Bernal-García et al. [34] } & \multirow{2}{*}{$\operatorname{MDEA}(1)+\mathrm{H}_{2} \mathrm{O}(2)$} & $w_{1}=0.3 ; 0.4$ \\
\hline & & $T=(313.15-353.15) \mathrm{K}$ \\
\hline \multirow{4}{*}{ Maham et al. [35] } & \multirow{4}{*}{$\operatorname{MEA}(1)+\mathrm{H}_{2} \mathrm{O}(2)$} & $w_{1}=0.1 ; 0.2 ; 0.3 ; 0.4$ \\
\hline & & $T=(313.15-333.15) \mathrm{K}$ \\
\hline & & $w_{1}=0.3 ; 0.4$ \\
\hline & & $T=353.15 \mathrm{~K}$ \\
\hline
\end{tabular}


Percentage deviations from those reference values are plotted in figure 5 as a function of viscosity. Uncertainties, which are represented by the two converging lines, are considered to vary linearly between the values $\pm 2.4 \%$ and $\pm 2.9 \%$ shown before.

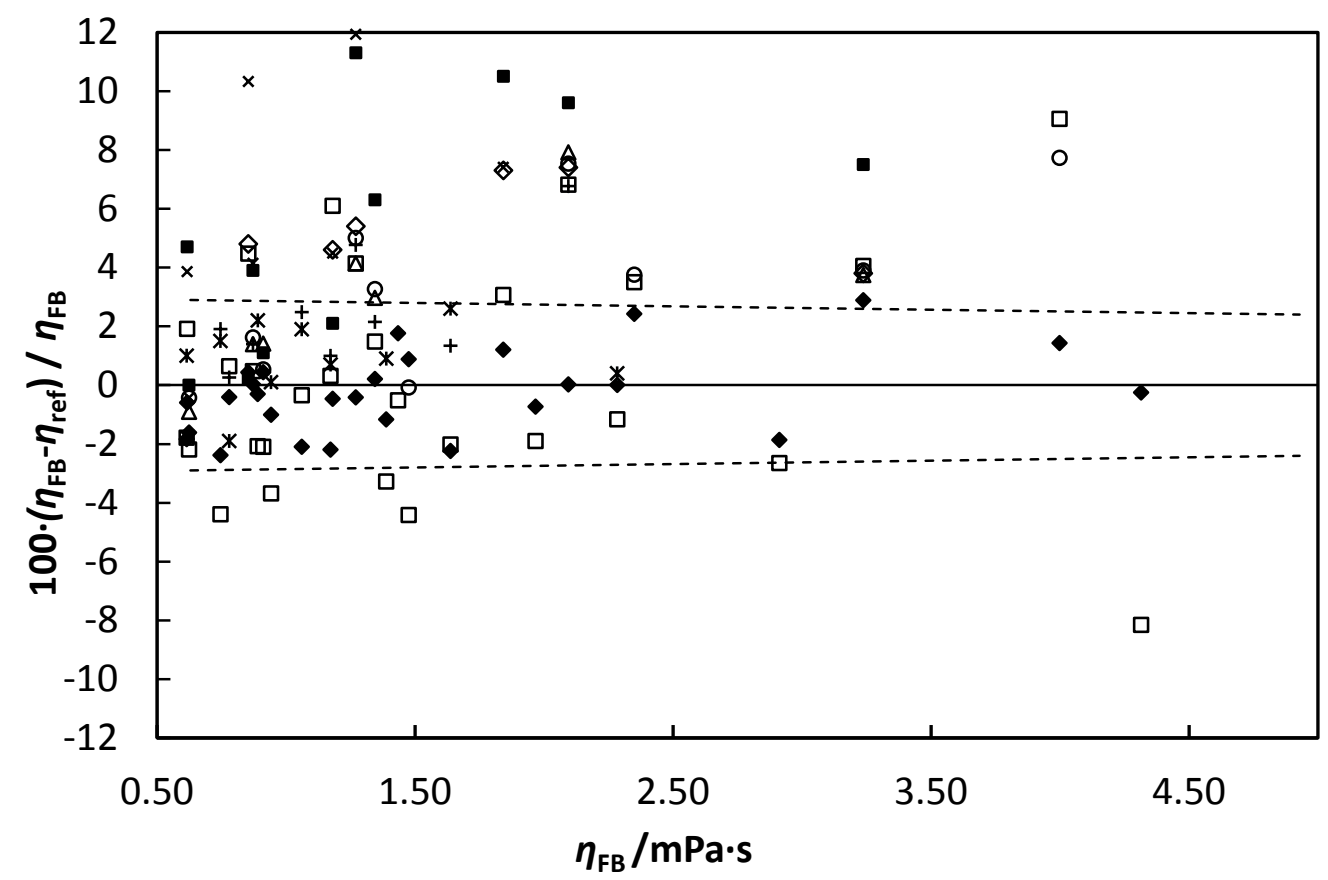

Figure 5. Relative deviation (\%) of the experimental data of MDEA $+\mathrm{H}_{2} \mathrm{O}$ and $\mathrm{MEA}+\mathrm{H}_{2} \mathrm{O}$ dynamic viscosity from the literature as a function of viscosity: Stabinger SVM 3000; $\times$ Rinker et al. [19]; ○ Al-Ghawas et al. [20]; + Li et al. [22]; $\Delta$ Paul et al. [27]; $\square$ Aranchchige et al. [32]; - Teng et al. [33]; $\diamond$ Bernal-García et al. [34] and * Maham et al. [35]. Lines represent the relative expanded uncertainty of our measurements.

Figure 5 shows a certain scattering among all data, which might be justified due to the intrinsic difficulty of measuring viscosity. In this sense, it is not possible to analyze quantitatively the compatibility of our data with others from the literature because most of literature values do not provide their associated uncertainties according to JCGM guide. However, knowing the uncertainties of both the falling body and the Stabinger SVM 3000 viscometers has made it possible to evidence the compatibility of their results. For MEA 
solutions, the best results were obtained in comparison with Maham et al. [30] with an average relative deviation of $1.3 \%$ (ten points for comparison) and these values were $3.4 \%$ in comparison with Al-Ghawas et al. [20] (ten points for comparison) and $3.8 \%$ with Aranchchige et al. [32] (fourteen points), for MDEA solutions.

\section{Conclusions}

Viscosity and density measurements of MDEA $+\mathrm{H}_{2} \mathrm{O}$ and MEA $+\mathrm{H}_{2} \mathrm{O}$ mixtures $(10 \%, 20 \%$, $30 \%$ and $40 \%$ amine mass fraction), at wide pressure and temperature ranges are presented. A recently developed falling body viscometer, able to measure viscosities of liquids from 0.1 $\mathrm{MPa}$ to $140 \mathrm{MPa}$ and from $253.15 \mathrm{~K}$ to $523.15 \mathrm{~K}$, is used in this work. It has been calibrated with $n$-dodecane and 1-butanol in a temperature range from $293.15 \mathrm{~K}$ to $353.15 \mathrm{~K}$ and pressures up to $120 \mathrm{MPa}$, obtaining a calibration range $\eta=(0.630$ to 4.929$) \mathrm{mPa} \cdot \mathrm{s}$. A detailed study of uncertainties was carried out, obtaining relative expanded uncertainties $(k=2)$ between $\pm 2.4 \%(4.954 \mathrm{mPa} \cdot \mathrm{s})$ and $\pm 2.9 \%(0.617 \mathrm{mPa} \cdot \mathrm{s})$.

In order to obtain said viscosities, densities were needed. These were obtained with an Anton Paar DMA HPM densimeter with an associated expanded uncertainty $(k=2)$ of $\pm 0.7 \mathrm{~kg} / \mathrm{m}^{3}$. Density and viscosity measurements were fitted using modified Tamman-Tait and VFT equations, respectively, obtaining standard deviations better than uncertainty measurements. Finally, the agreement of viscosity results of the falling body viscometer and Stabinger SVM 3000 at atmospheric pressure has been shown, and is completed with an exhaustive comparison to literature data. Both properties for MDEA solutions are always higher than for of MEA solutions under the same conditions. They decrease significantly when temperature increases and increase with pressure. Changes with temperature and pressure are quite similar for both amines although changes due to amine composition are more relevant for MDEA solutions. 


\section{Acknowledgments}

The authors M.S. and E.I.C. thanks to Education Ministry (Spanish Government) through a FPU scholarship and to Project for European Latin American Cooperation and Exchange (PEACE), respectively, for doctoral studies. The work was funded by the Regional Government of Castilla y León through the Project VA295U14.

\section{References}

[1] G.F. Versteeg, W.P.M. Van Swaaij, Chem. Eng. Sci. 43 (1988) 587-591.

[2] R.J. Little, W.P.M. Van Swaaij, G.F. Versteeg, AIChE J. 36 (1990) 1633-1640.

[3] A. Veawab, A. Aroonwilas, P. Tintiwachwuthikul, ACS Division of Fuel Chemistry Preprints 47 (2002) 49-50.

[4] D. Aaron, C. Tsouris, Separation Sci. \& Tech. 40 (2005) 321-348.

[5] C. Hsu, M. Li, J. Chem. Eng. Data 42 (1997) 502-507.

[6] J.J. Segovia, O. Fandiño, E.R. López, L. Lugo, M.C. Martín, J. Fernández, J. Chem. Thermodyn. 41 (2009) 632-638.

[7] J.R. Zambrano, M. Sobrino, M.C. Martín, M.A. Villamañán, C.R. Chamorro, J.J. Segovia, J. Chem. Thermodyn. 96 (2016) 104-116.

[8] Evaluation of measurement data - Guide to the expression of uncertainty in measurement. JCGM 2008.

[9] P. Daugé, A. Baylaucq, L. Marlin, C. Boned, J. Chem. Eng. Data 46 (2001) 823-830.

[10] W.A. Wakeham, A. Nagashima, J.V. Sengers, Experimental Thermodynamics, Vol. III: Measurement of the Transport Properties of Fluids, Blackwell Scientific Publications, Oxford, 1991.

[11]M. Zeng, C.J. Schaschke, Int. J. Chem. Eng. (2009) 1-8. 
[12]M.J.P. Comuñas, X. Paredes, F.M. Gaciño, J. Fernández, J.P. Bazile, C. Boned, J.L.

Daridon, G. Galliero, J. Pauly, K.R. Harris, J. Chem. Thermodyn. 69 (2014) 201-208.

[13] J.R. Zambrano, M. Sobrino, M.C. Martín, M.A. Villamañán, C.R. Chamorro, J.J.

Segovia, J. Chem. Thermodyn. (2016) http://dx.doi.org/10.1016/j.jct.2015.12.021.

[14] M. Sobrino, J.J. Segovia, Dyna 87 (2012) 438-445.

[15]M.C.S. Chen, J.A. Lescarboura, G.W. Swift, AIChE J. 14 (1968) 123-127.

[16]C.J. Schaschke, S. Allio, E. Holmberg, Food Bioprod. Process. 84 (2006) 173-178.

[17]M. Sobrino, Desarrollo de un viscosímetro para la caracterización a alta presión de nuevos biocombustibles y mezclas acuosas de aminas para la captura de $\mathrm{CO}_{2}, \mathrm{PhD}$ Thesis, University of Valladolid, Spain, 2015.

[18]D.R. Caudwell, J.P. Trusler, V. Vesovic, W.A. Wakeham, International Journal of Thermophysics 25 (2004) 1339-1352.

[19]E.B. Rinker, D.W. Oelschlager, A.T. Colussi, K.R. Henry, O.C. Sandall, J. Chem. Eng. Data 39 (1994) 392-395.

[20]H.A. Al-Ghawas, D.P. Hagewlesche, G. Ruiz-Ibanez, O.C. Sandall, J. Chem. Eng. Data 34 (1989) 385-391.

[21] M. Li, K. Shen, J. Chem. Eng. Data 37 (1992) 288-290.

[22] M. Li, Y. Lie, J. Chem. Eng. Data 39 (1994) 444-447.

[23] J.M. Bernal-García, M. Ramos-Estrada, G.A. Iglesias-Silva, K.R. Hall, J. Chem. Eng. Data 48 (2003) 1442-1445.

[24] J. Han, J. Jing, D.A. Eimer, M.C. Melaaen, J. Chem. Eng. Data 57 (2012) 1843-1850.

[25] F. Pouryousefi, R.O. Idem, Ind. Eng. Chem. Res. 47 (2008) 1268-1276.

[26] A. Zúñiga-Moreno, L.A. Galicia-Luna, J.M. Bernal-García, G.A. Iglesias-Silva, J. Chem. Eng. Data 52 (2007) 1988-1995.

[27] S. Paul, B. Mandal, J. Chem. Eng. Data 51 (2006) 1808-1810. 
[28]Y.M. Tseng, R. Thompson J. Chem. Eng. Data 9 (1964) 264-267.

[29] T.G. Amundsen, L.E. Øi, D.A. Eimer J. Chem. Eng. Data 54 (2009) 3096-3100.

[30] J. Han, J. Jing, D.A. Eimer, M.C. Melaaen, J. Chem. Eng. Data 57 (2012) 1095-1103.

[31] M.J.P.Comuñas, A. Baylaucq, C. Boned, J. Fernández, Int. J. Thermophys. 22 (2001) 749-768.

[32] U.S.P.R. Arachchige, N. Aryal, D.A. Eimer, M.C. Melaaen, Annual Transact. Nordic Rheology Soc. 21 (2013) 299-306.

[33] T.T. Tseng, Y. Maham, L.G. Hepler, A.E. Mather, J. Chem. Eng. Data 39 (1994) 290293.

[34] J.M. Bernal-García, L.A. Galicia-Luna, K.R. Hall, M. Ramos-Estrada, G.A. IglesiasSilva, J. Chem. Eng. Data 49 (2004) 864-866.

[35] Y. Maham, C.N. Liew, A.E. Mather, J. Sol. Chem. 31 (2002) 743-756. 